\title{
Immunolocalization of E-cadherin and beta- catenin in the cyclic and early pregnant canine endometrium
}

\author{
R. Payan-Carreira, M. A. Pires, C. Santos, B. Strom Holst, J. Colaco and Heriberto \\ Rodriguez-Martinez
}

Journal Article

\section{Tweet}

N.B.: When citing this work, cite the original article.

Original Publication:

R. Payan-Carreira, M. A. Pires, C. Santos, B. Strom Holst, J. Colaco and Heriberto RodriguezMartinez, Immunolocalization of E-cadherin and beta-catenin in the cyclic and early pregnant canine endometrium, Theriogenology, 2016. 86(4), pp.1092-1101.

http://dx.doi.org/10.1016/j.theriogenology.2016.03.041

Copyright: Elsevier

http://www.elsevier.com/

Postprint available at: Linköping University Electronic Press

http://urn.kb.se/resolve?urn=urn:nbn:se:liu:diva-131682
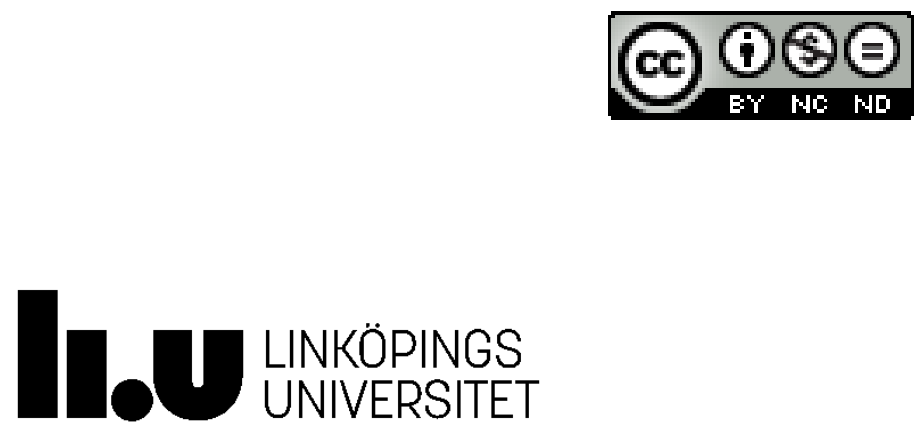
IMMUNOLOCALIZATION OF E-CADHERIN AND $\beta$-CATENIN IN THE CYCLIC AND EARLY PREGNANT CANINE ENDOMETRIUM

R. Payan-Carreira ${ }^{a \star}$, M. A. Pires ${ }^{a}$, C. Santos ${ }^{b}$, B. Ström Holst ${ }^{c}$, J. Colaço ${ }^{a}$; H. RodriguezMartine $^{\mathrm{d}}$

a CECAV, University of Trás-os-Montes and Alto Douro, P.O. Box 1013, 5001-801 Vila Real, Portugal.

b DEBA, University of Trás-os-Montes and Alto Douro, P.O. Box 1013, 5001-801 Vila Real, Portugal.

c Dept. of Clinical Sciences, Box 7054, Swedish University of Agricultural Sciences (SLU), 75007 Uppsala, Sweden

${ }^{\mathrm{d}}$ Dept of Clinical and Experimental Medicine, Faculty of Medicine \& Health Sciences, Linköping University, 58185 Linköping, Sweden

* Corresponding author: Rita Payan-Carreira, CECAV, Department of Zootecnics, Universidade de Trás-os-Montes e Alto Douro; Vila Real, Portugal; Fax: +351.259350480 . E-mail address: rtpayan@gmail.com 


\begin{abstract}
Putative changes in E-cadherin and $\beta$-catenin during implantation in dogs are of interest to study, as they are both relevant for epithelial integrity. E-cadherin and $\beta$-catenin were immunolocalised in the canine cyclic and early pregnant endometrium, using monoclonal antibodies. Both molecules were detected in all types of endometrial epithelia (surface, superficial glandular and deep glandular epithelia) at all stages of the estrous cycle and in early placental structures. E-cadherin depicted a gradient of immunoreaction, intensity apparently being lowest in the surface epithelium to progressively increase towards the deepness of the endometrial glands, regardless of the stage of estrous cycle. The overall immunostaining was, however, weaker at diestrus. In pregnant samples trophoblast immunolabeling was stronger than in the endometrial surface epithelium; in the latter, the cytoplasmic pattern predominated over the membranous, as was also seen in the giant decidual cells in the labyrinth. In the early placenta, only trophoblast cells and lacunae retained membrane signals. $\beta$-catenin membrane labeling appeared relatively constant through the cycle, although a tendency towards a decrease in intensity was detected at the secretory stages. In addition, a dislocation of the immunoreaction to the cytoplasm was observed in both the surface and the glandular epithelia in particular stages of the cycle. In early pregnancy, a loss of the membranous pattern was observed in the surface epithelium and labyrinth, but neither on trophoblast nor in lacunae. The results showed the existence of a softening of adherens junctional complex in progestagen-dominated stages favoring embryo-maternal interactions and endometrial invasion during implantation.
\end{abstract}

Key words: E-cadherin; $\beta$-catenin; canine endometrium; estrous cycle; immunohistochemistry; dog. 


\section{Introduction}

In the bitch, like in other mammals, the endometrium consists of a mesenchymal stroma, composed of individual fusiform cells surrounded by extracellular matrix, and two epithelial cell populations: the glandular epithelium and the surface or luminal epithelium (SE). The glandular epithelium is usually further classified into superficial glandular epithelium (SGE), located at the crypt zone, and deep glandular epithelium (DGE), located at the basal zone of the endometrium [1]. Both epithelial types are secretory and of utmost relevance for the integrity of the organ, for the interactions with the spermatozoa and with the embryo, and for the establishment and maintenance of pregnancy. A complex sequence of signaling events, encompassed by the crosstalk between endometrial epithelium and stroma, is crucial to the establishment of pregnancy [2, 3].

In response to cyclic variations in sex hormones, the mammalian endometrium evidences recurrent structural and morphological modifications in what is frequently named the endometrial cycle [4]. Several molecules have been shown to display cyclic patterns of expression, responding differently to the dominance by estrogen or progesterone concentrations on target tissues, contributing to normal functioning of the endometrium and the endometrial receptivity to the embryo [4-7]. The endometrial remodeling processes, controlled by sex steroids, entail complex changes in epithelial cell-cell interactions, which are required to maintain the continuity and integrity of the endometrium [8].

In the endometrium, the epithelial lining creates a protective barrier against bacterial or viral pathogens as well as against different antigens while in particular periods, such as at the window of implantation, it displays a permissive status that allows embryo-maternal interactions, culminating with the establishment of placenta. Adhesive interactions between neighboring epithelial cells are also crucial for tissue morphogenesis and renewal [9]. 
Endometrial modifications in the expression of different adhesion molecules during the cycle or early pregnancy have been reported in humans [8, 10-12], sheep [13] and pigs [12, 14]. Adherent junctions play important functions in the maintenance of intercellular connections, which defines the characteristic architecture of the epithelial lining. Through cadherinmediated epithelial cell-cell connections, the integrity and strength of the epithelial layer [15, 16], as well as the polarity of the epithelia [17] are maintained. Consequently it can be presumed that the plasticity of cell-to-cell adhesion of endometrial epithelial cells is crucial to female fertility.

E-cadherin, a member of the cadherin family of calcium-dependent adhesion molecules, is a cell surface glycoprotein and an important determinant of tissue processes related to selective cell adhesion or detachment $[15,16]$, which in the uterus involves changes designed to support the implantation and growth of an embryo $[8,18,19]$.

The cyclic tissue remodeling that occurs in the endometrium during the cycle requires complex changes in cell-cell and cell-matrix interactions. Spatiotemporal changes in the Ecadherin adhesion system may be associated with the morphologic changes observed in the mammals' endometrial cycle, a dynamic process that comprises epithelial cell rearrangements, proliferation, renewal and movement that involves the permanent breaking and reforming of cell-cell adhesions [10, 20]. Furthermore, down-regulation of E-cadherin has been associated to invasive processes, which in the uterus includes the disruption of the epithelial barrier and the progression through a permissive extracellular mesenchymal matrix, as occurs at implantation in species with decidual placenta [21]. Supporting this concept, Dawood et al. [22] showed that E-cadherin and its gene transcripts were expressed in peri-implantation phase endometrium in women and Liu et al. [23] found a relationship between E-cadherin and metalloproteinase-2 and -9 during the mouse embryo implantation process. As endometrial invasion is a tightly controlled process, E-cadherin expression at 
the window of implantation could be associated to the regulation of the migrating and invasive potential of the trophoblast. It was demonstrated that E-cadherin expression is significantly reduced close to the maternal recognition period in sheep [13] and pigs [14], species where it may also play a favorable role in embryo elongation. In dogs, Guo and collaborators [24], using in situ hybridization, described a reduction in E-cadherin mRNA expression until day 20 of pregnancy compared with the expression levels recorded in estrus, thereafter showing a strong signal in the glandular epithelium. Based on these findings the authors suggest a role for this molecule in the canine implantation process.

The E-cadherin junctional complex also includes several other molecules, such as $\beta$-catenin, by which the cadherin molecule is anchored to the cytoskeleton. In the cytoplasm, $\beta$-catenin pairs bind strongly to the E-cadherin domain, binding the complex to the actin skeleton through the $\alpha$-actin molecule [25-27]. In addition to its role in cell-to-cell adhesion, $\beta$-catenin also plays an important role in the Wnt/Wg growth factor signaling pathway [28, 29], which has been implicated in the regulation of the endometrial cycle [30] and in implantation [31]. $\beta$-catenin participation in either cell adhesion or the Wnt pathway depends on the existence of a competitive binding of this molecule to E-cadherin and Wnt-signaling molecules, which is determined by the activity of different kinases [28, 29, 31].

Epithelial functional or physical integrity is a major issue regarding embryo invasion in early pregnancy. In dogs, the process of embryo implantation involves embryonic adhesion to the maternal epithelium, quickly followed by trophoblast invasion and the concurrent erosion and transformation of the upper half of the endometrium to establish an endotheliochorial placenta $[2,32]$. Thereby, changes in the E-cadherin/ $\beta$-catenin adhesive complex are expected to occur at the initial moments of implantation in dogs. Disgregation of components of this complex will result in lateral cell-cell dissociation [2, 33], which would facilitate embryo invasion, as it occurs during tumour progression [34, 35]. A reduced endometrial epithelial 
barrier, as indicated by a reduction in E-cadherin expression or the compromised polarity of epithelia during the luteal or secretory stage might also favor the development of pyometra during progesterone dominance, facilitating the colonization of the endometrium by pathogen bacteria ascending from the vagina [36]. This risk is likely higher in species with a physiologically long dioestrus, like dogs.

Despite extensive research reported on cadherin and $\beta$-catenin activity in the uterus of different species $[8,13,18,37]$, information about the immunolocalization of E-cadherin and $\beta$-catenin in the canine endometrium is sparse. Canine reproductive physiology depicts several characteristics distinguishing this species from other domestic animals, particularly non-carnivorous. Of particular interest is the relatively long estrous phase and a remarkable long luteal phase which is of similar length in pregnant and non-pregnant cycles [38]. The dog presents an endotheliochorial placenta type, where the invasive trophoblast destroys the luminal epithelium and the underlying lamina propria in the process of implantation and placentation $[39,40]$. Consequently, the spatiotemporal pattern in E-cadherin and $\beta$-catenin molecules in the canine endometrium may differ from those established in other species with a shallower non-invasive placentation. Considering these concepts, the aim of this work was: $1 /$ to analyze E-cadherin and $\beta$-catenin protein immunolocalization throughout the stages of the canine estrous cycle and to determine whether temporal changes exist during the uterine cycle and to; $2 /$ ascertain possible modifications of cadherin/ $\beta$-catenin adhesion pathways in the canine embryo apposition and adhesion to the endometrial lining epithelium.

\section{Material and methods}

\section{Animals}

A total of 50 mature, healthy bitches, submitted to elective ovariohysterectomy were used. Additionally nine pregnancy samples were obtained from females with unwanted 3 weeklong pregnancies, submitted to elective ovariohysterectomy. Before surgery, a vaginal 
cytological specimen was obtained. A blood sample was collected by venipuncture from a jugular vein to a controlled vacuum tube (Serum-gel, S-Monovette ${ }^{\circledR}$, Sarstedt, Germany), and promptly centrifuged at $2,500 \mathrm{~g}$ for 15 minutes. The serum was stored at $-20^{\circ} \mathrm{C}$ until analysis. Serum progesterone levels were determined by a chemiluminescent immunoassay system (Immulite ${ }^{\circledR}$, DPC-Diagnostic Products Corp., Los Angeles, CA, USA).

Endometrial samples were collected after the expressed consent of the animals' owners, and in respect to the International Ethical standards. Excised genital tracts were fixed in 10\% buffered formalin solution immediately after surgery, for no more than 3 days. A section from each uterine horn was collected at its caudal ending, at approximately $1 \mathrm{~cm}$ above the uterine body, and thereafter embedded in paraffin wax, sectioned at $3 \mu \mathrm{m}$ thickness and routinely stained with haematoxylin and eosin for histological staging of the estrous cycle and for excluding uterine pathologies. In this study, only one of the uterine samples was used.

\section{Staging of the estrous cycle and early pregnancy}

The staging of the estrous cycle was performed after the genital tract collection, at the laboratory, based on the cumulative information provided by vaginal cytology, inspection of the ovaries at $\mathrm{OVH}$, and circulating levels of progesterone. Vaginal cytology allowed a preliminary staging of the cycle and was particularly useful to distinguish proestrus and estrus. The ovary and uterine morphology and the serum progesterone were used for further staging, as previously described $[41,42]$. Uterine samples for the proestrus $(n=8)$, estrus $(n=10)$, early diestrus $(n=12)$, full diestrus $(n=10)$ and anestrus $(n=10)$ were used in this study. 
Nine samples representing pregnancy days 17 to 20 after the LH-surge were selected. The pregnancy samples were staged on the basis of cumulative information gathered from a diestrus-compatible cytology, the date of documented coitus plus the histological changes observed in the endometrium, according to the histological descriptions of canine early pregnancy events $[39,43]$, and aligned to the day from pre-ovulatory LH surge. Up to day 17 the embryo was not yet adhered to the endometrium, although some changes are found in the superficial layers of the endometrium, including increased interstitial edema and deepening of the endometrial crypts. On day 17 , the trophoblast grew and wedged the maternal surface epithelium (SE). Only small lacunae were visible. Between days 18 and 20, the embryo adherence prompted endometrial invasion; the trophoblast continued to spread down, and the syncytial cells to penetrate deeper in the endometrium forming the labyrinth that appeared as strong, linear, closely packed cords, often presenting mitotic figures. Until this moment, a limited development of the basal glands was observed.

\section{Immunohistochemistry (IHC)}

IHC-analysis was performed on tissue sections $3 \mu \mathrm{m}$ thick on silane-coated slides and a streptavidin-biotin-peroxidase technique (UltraVision, Lab Vision, Fremont, CA, USA). The primary antibodies used were mouse monoclonal antibodies raised against E-cadherin (Clone 4A2C7, Invitrogen) and $\beta$-catenin (Clone CAT-5H10, Invitrogen), at 1:100 dilution in PBS, as previously established for canine tissues [44].

The slides were submitted to routine de-paraffinization and rehydration in graded alcohol, and pre-treated to enhance antigen retrieval (microwave irradiation; $750 \mathrm{~W}$, three cycles of 5 min, with slides immersed in a $0.05 \%$ Extran solution); thereafter, the endogenous peroxidases were blocked by 30 minutes immersion in 3\% hydrogen peroxide/PBS, followed by inhibition of non-specific binding by incubation with Large Volume Ultra V-Block (UltraVision, Lab Vision, Fremont, CA, USA) for $5 \mathrm{~min}$. Incubation with primary antibodies was performed in a humid chamber, overnight at $4^{\circ} \mathrm{C}$. Afterwards, the labeled slides were 
incubated with a biotin conjugated secondary antibody, followed by incubation with enzymelabeled streptavidin, for 10 minutes each (Biotinylated Goat Polyvalent Plus $®$ antibody and Streptavidin-peroxidase Plus ${ }^{\circledR}$ - UltraVision, Lab Vision, Fremont, CA, USA - respectively), at room temperature. The reactions were visualized using DAB (3,3'-diaminobenzidine) as a chromogen, and the slides were counterstained with haematoxylin, dehydrated and mounted for light microscopy evaluation.

For negative controls, primary antibodies were replaced by either the normal mouse IgG (sc2025; Santa Cruz Biotechnology, Inc., Europe, Heidelberg, Germany) or PBS. In neither negative control, immunoreaction against the two molecules was detected. Samples from a canine mammary carcinoma were used as positive controls.

\section{Immunohistochemical scoring}

Two independent observers blindly assessed the immunoreaction for E-cadherin and $\beta$ catenin, in a NIKON Eclipse 80i (Nikon Instruments Europe, BV) photomicroscope. The distribution of E-cadherin and $\beta$-catenin immunoreaction was studied for each of the endometrial epithelial types: the surface epithelium (SE), the superficial glandular epithelium (SGE) and the deep glandular epithelium (DGE). Positivity was indicated by the presence of a distinct brown membrane labeling. For both molecules, the evidence of a membrane staining was semi-quantitatively scored using a 4-point scale: weak (1), moderate (2), strong (3) or very strong (4).

Cytoplasmic staining was frequently seen in addition to membranous staining for both molecules. For E-cadherin, membrane labeling extending to the cytoplasm was frequently found; it has been considered to correspond to its cytoplasmic domain and it was not scored independently whenever it followed membrane labeling. When cells failed to show membrane immunoreaction, and immunoreaction was located exclusively in the cytoplasm, the pattern was scored as cytoplasmic.

For $\beta$-catenin, the membrane dislocation of the immunoreaction was assessed due to its role at the cell-to-cell junctions and at the $\mathrm{Wnt} / \mathrm{Wg}$ signaling pathways. The subcellular pattern 
and the differences in the membrane and cytoplasmic staining were scored independently for intensity and pattern, the latter being further scored as diffuse, scattered (or dot-like), apical or supra-nuclear.

The immunoreaction in pregnancy samples was graded using the same semi-quantitative scoring system, which was applied individually for the trophoblast, the surface epithelium and the epithelium of the labyrinth (or syncytium cords) and the lacunae, as well as for the deep endometrial glands.

\section{Statistical analysis}

Statistical comparisons were performed by using the statistical software IBM SPSS Statistics version 22.0 for Mac OS X 10.8. Associations between the intensity and patterns of immunoreaction for E-cadherin and $\beta$-catenin and the categorical variables (stage of the estrous cycle and epithelial type) were performed using the chi-square and Fisher exact tests. The Z-test was performed for group comparisons, the P-values adjusted by the Bonferroni method. Comparisons between early diestrus and pregnancy samples were only established for the surface and the deep glandular epithelia. A $P$ value $\leq 0.05$ was regarded as statistically significant.

\section{Results}

\section{The non-pregnant canine endometrium}

The scoring results for the IHC-distribution of E-cadherin and $\beta$-catenin in the non-pregnant endometrium are shown in Figures 1 to 3 as well as in Tables 1 and 2. For both molecules, a typical membranous labeling was evident in all samples and in all the epithelial types. Changes of the intensity of the immunolabeling were detected during the estrous cycle (Figure 1). In addition to the membrane immunolabeling, different cytoplasmic patterns of immunolabeling were observed for $\beta$-catenin, and scored accordingly (Table 2). 
In general, the E-cadherin immunolabeling showed a gradient of intensity between the different epithelial types $(P<0.001$; Fisher= 115.376); it was lower in the superficial epithelium and progressively increasing towards the deep endometrial glands (Figure 2).

When studied individually, the different endometrial epithelial types tended to show differences in E-cadherin membrane intensity of immunolabeling during the different stages of the estrous cycle. The SE showed similar immunostaining scores in anestrus, proestrus and estrus, evidencing a moderate immunopositivity (Figure 2; Table 1). In contrast, during early diestrus and diestrus (Figures 1.E and 2), a clear reduction of the intensity of immunolabeling was noticed $(P<0.001 ;$ Fisher $=50.681)$. For the SGE, a stronger immunoreaction was detected in anestrus and proestrus (Figures 1.A and 2), which tended to decrease in estrus (Figure 1.C). A reduction of the intensity of the immunolabeling $(\mathrm{P}<0.001 ;$ Fisher $=42.224)$ was recorded in early diestrus and diestrus (Figures $1 . \mathrm{F}$ and 2$)$. The intensity of the DGE immunostaining was found to be similar between anestrus and proestrus (Figure 2), with epithelial cells evidencing a very strong labeling for E-cadherin. In estrus, a tendency for a decrease in the intensity of labeling was found (Figure 1), with a clear decrease in early diestrus and diestrus (Figures 1.G and 2) $(P<0.001$; Fisher $=$ 61.143), most obvious in early diestrus. Overall, stronger immunolabeling for E-cadherin was observed during anestrus, proestrus and estrus compared with early diestrus and diestrus $(P<0.001 ;$ Fisher = 154.265) $($ Table 1; Figure 2). When the variations in the intensity score for all the three epithelial types were evaluated together, a significant difference was found between the different stages of the canine estrous cycle $(P<0.001$; Fisher $=154.265)$ with a decrease of the E-cadherin immunoreaction in the progesterone-mediated stages (early diestrus and diestrus).

The membrane intensity scores for $\beta$-catenin varied between low to moderate for all the endometrial epithelia (Figure 1.B and Figure 3; Table 1). In general, the type of epithelium 
influenced the intensity of $\beta$-catenin immunolabeling $(P<0.001$; Fisher $=63.989)$ : a tendency for lower intensity scores in SE and SGE was observed, as for E-cadherin, whilst the DGE tended to present stronger intensity scores. However, no significant changes in the membrane staining intensity during the cycle stages were found within epithelial types $(P=$ 0.901; Fisher = 12.676)

Diverse cytoplasmic immunoreactivity was observed for $\beta$-catenin (Figure 4; Table 2). Overall, cytoplasmic immunoreaction had lower intensity scores in early diestrus and in diestrus, compared to anestrus, proestrus or estrus $(P<0.001$; Fisher $=44.311)$. In addition, the cytoplasmic pattern was diffuse in all cycle stages with exception for estrus, when the supra-nuclear pattern predominated in all the three epithelial types $(P<0.001$; Fisher $=$ 108.770). The supra-nuclear and apical patterns (Figures 1.D and 1.F) usually co-existed with higher cytoplasmic intensity scores $(P=0.006$; Fisher $=19.309)$.

$\beta$-catenin cytoplasmic immunolabeling during the canine estrous cycle presented a significant association between immunostaining and epithelial type $(P<0.001$; Fisher $=$ 67.749) as well as between the cytoplasmic intensity and pattern $(P<0.001$; Fisher $=$ 108.770). The SE had a low intensity cytoplasmic immunostaining in early and mid diestrus, and a moderate intensity in all other stages (Figure 4). In both diestrus stages the diffuse pattern dominated over the apical or the supra-nuclear areas (Figure 5). The SGE showed high membrane and cytoplasmic intensity scores in all cycle stages except during dioestrus. A diffuse cytoplasmic pattern was found in anestrus and proestrus, while in diestrus the diffuse and apical patterns were equally common; a scattered cytoplasmic pattern (Figure 1.H) was the most prevalent pattern in early diestrus samples, while in estrus the supranuclear staining (Figure 5) was the most frequently observed. In DGE, the intensity of the cytoplasmic immunostaining did not vary between cycle stages with exception of estrus, when higher intensity scores were observed. A diffuse cytoplasmic pattern of labeling was found predominantly during anestrus and proestrus (Figure 5); the supra-nuclear pattern 
predominating during estrus while in early diestrus (Figure 1.H) the scattered cytoplasmic pattern prevailed over the apical location pattern (50\% Vs. $25 \%$ of the samples). In diestrus the apical (Figure 1.B) and diffuse patterns prevailed.

Intensity of $\beta$-catenin membrane and cytoplasmic staining was significantly lower in progesterone associated-stages of the estrous cycle $(P<0.001$, Fisher $=16.112$ and $P<$ 0.001, Fisher $=60.245$, respectively). Furthermore, the supranuclear pattern of strong intensity was consistently found in the cycle stage where estrogens peak $(P=0.003$; Fisher $=25.661)$. A significant association between the intensity of the membrane immunolabeling for $E$-cadherin and for $\beta$-catenin was found $(P<0.001$; Fisher $=274.471)$.

\section{The early pregnant canine endometrium}

Table 3 summarizes the information concerning the E-cadherin and $\beta$-catenin immunolabeling in the canine pregnant endometrium (days 17 to 20).

The trophoblast showed a strong or very-strong E-cadherin immunolabeling (Figure 6.A; Table 3), in contrast to the maternal SE that predominantly showed a weak immunostaining and often failed to evidence a membrane pattern for this molecule (Figure 6.A; Table 3). No differences were observed in the immunoreaction for E-cadherin in the SE in early pregnant and early diestrus stage.

The E-cadherin membranous pattern was also heterogeneously presented in the labyrinth: the trophoblast population displayed a moderate to strong membrane pattern (Figure 6.A), whereas in the giant decidual cells the membrane pattern was absent and a weak cytoplasmic pattern was noticed (Figure 6.A and 6.C). When lacunae were formed, closer to pregnancy day 20, the membrane immunolabeling for E-cadherin was strong (Figure 6.E). No differences were found for DGE intensity scores between early pregnant and early diestrus stage (Tables 1 and 3); in both the stages a moderate intensity of immunolabeling was observed. 
Considering the immunostaining for $\beta$-catenin, in the epithelial types represented in the early pregnant canine endometrium the moderate intensity score prevailed (Table 3). The trophoblast showed a moderate membrane intensity (Figure 6.B), which is also represented in the trophoblast populations located in the labyrinth cords. Contrasting, the giant decidual cells were negative for $\beta$-catenin or displayed a weak, cytoplasmic labeling (Figure 6.D). The intensity of the immunolabeling for this molecule in DGE was not different from the described for the early diestrus stage (Tables 1 and 3); the most represented intensity score was the moderate.

\section{Discussion}

Previous work in human and rodents showed that adhesion signaling molecules change in the endometrium during the estrous cycle in response to the sex steroid dynamics $[10,19$, 37]. Moreover, it has been proposed that E-cadherin/catenin complexes may play a role in initial attachment of the embryo during implantation [45], besides the maintenance of the endometrial histoarchitecture [37].

Results from the present study clearly show the existence of differences in the intensity or pattern for E-cadherin and $\beta$-catenin immunolabeling according to changes in the sex steroid milieu. The immunostaining was evidenced for both molecules in all canine endometrial samples and on all the epithelial elements (SE, SGE and DGE). E-cadherin was abundantly expressed in anestrus and in the proliferative stages of the bitch estrous cycle (proestrus and estrus), but its immunolabeling decreased during progesterone dominance, in particular in full diestrus, when progesterone levels are high. A similar down-regulation of E-cadherin expression during the progesterone phase of the cycle was reported in ewes [13] and in humans $[37,45]$. However, a small increase in E-cadherin mRNA was observed following exogenous progesterone administration to ovariectomized bitches [24]. This slight upregulation of E-cadherin mRNA, which seems to contradict the immunohistochemical results 
presented in the present study, might be associated to the acute administration of progesterone upon a non sex steroid-primed endometrium, while in the present report, uterine samples from normally cycling bitches were used. The acute progesterone administration could induce a down-regulation of the endometrial progesterone receptors, mimicking a late diestrus phase. On the other hand, that study focused on the activation of the E-cadherin synthesis pathway, whereas the protein was evidenced in the experiment presented herein. Differences could also be explained by a transcription regulatory mechanism.

The present study revealed that $\beta$-catenin membrane immunolabeling changes during the canine estrous cycle, with a tendency to decrease to a lower expression during the secretory stages of the cycle (diestrus stages). Such changes significantly correlated with decreased immunohistochemical expression of E-cadherin in the membrane. This may suggest a reduction of the E-cadherin/ $\beta$-catenin complexes, which could be associated with a modification of the adherens junction properties, in order to facilitate embryo interaction with the endometrium. An apparent decrease in the intercellular junctional strength, that was more pronounced in the canine SE and the SGE, would allow trophoblast cells to migrate between the epithelial cells during implantation. Evidence collected from early pregnancy samples representing the adhesion and initial invasion steps of the implantation process supports that hypothesis. Loss of the membrane pattern and a rather weak intensity of Ecadherin immunoreaction in the SE was seen during trophoblast invasion. Concomitantly, an exclusive diffuse cytoplasmic pattern for $\beta$-catenin immunolabeling was seen. Adhesive intercellular interactions are crucial for endometrial epithelial differentiation [46]. Taken together, these findings support the loss of lateral cell-to-cell adherence, preceding the apposition and endometrial invasion of the canine embryo. The loss of E-cadherin/ $\beta$-catenin adherence at early pregnancy, as observed herein in the SE and in the giant decidual cells in the labyrinth, is suggestive of the existence of a transitional process in these cells, occurring during invasion and establishment of placenta in dogs. This is similar to changes 
proposed by Bartley and colleagues [47] to occur in human endometriosis, allowing endometrial cells to detach from their primary site and adhesion and invade the implantation sites to form endometriotic lesions.

For most mammalian species with invading trophoblast, embryo strategies towards implantation mimic those of tumour tissues $[34,35]$. Involvement of E-cadherin/ $\beta$-catenin complexes in invasion suppression has been widely proven, in particular concerning tumour progression and invasiveness. It has been demonstrated that a decrease in E-cadherin membrane expression and of E-cadherin/ßcatenin complexes, along with the existence of aberrant $\beta$-catenin expression, result in loss of the epithelial phenotype accompanying increased invasiveness and hence a higher metastatic ability [34, 35, 37, 48]. A decrease in E-cadherin membrane expression usually accompanies a weakened attachment to the cellular skeleton and destabilization of the cell-to-cell adhesion to each other [25, 27, 28]. In parallel with a decrease in its expression, an increase in the E-cadherin instability and degradation associated with a decrease in the active cadherin complex has been described $[26,28,49]$. Based on the findings reported in the current study, a comparable situation could be hypothesized to occur in the endometrial epithelia at embryo implantation, as in the present study a decrease in E-cadherin junctional complexes during progesteroneassociated stages and at early pregnancy was shown.

Another interesting concept that recently issued from oncology research is that loss of functional E-cadherin contributes to tumour cells resistance to apoptosis. This concept associates the loss of cadherin mediated adhesion complexes with a cellular mechanism that allows the cells to escape induced programed death mediated either by TRAIL $[50,51]$ or Bcl-2 [52], respectively associated to the extrinsic/TNF-mediated and the intrinsic apoptosis pathways. Such hypothesis cannot be ignored, as it also could be a plausible explanation for the atypical pattern immunostaining observed herein in cells that participate in the canine placental labyrinth. The regulation of apoptosis in the placental labyrinth could 
contribute to its remodelling by maintaining the syncytiotrophoblast:cytotrophoblast ratio or in more general terms the balance between foetal and maternal tissues.

As seen in the present study, a reduction in cadherin/catenin membrane expression during early and full diestrus may, by interfering with the strength of the epithelial endometrial barrier, also modulate the local susceptibility to invading pathogens and inflammation. Associated to a decrease in MUC1 expression occurring during canine diestrus [53], the reduction in the epithelial E-cadherin active complex might contribute to the onset of canine pyometra in diestrus.

Concerning the cytoplasmic immunostaining for $\beta$-catenin in the present study, a nuclear immunostaining for this molecule was not found, contradicting sporadic nuclear immunolabeling reported in humans $[37,54]$. In the uterus, cytoplasmic $\beta$-catenin expression should not be considered abnormal, even when nuclear expression is detected, as this molecule is involved in cellular pathways other than the E-cadherin junctional complexes [8] The Wnt/Wg signaling pathway has been demonstrated to be present in the endometrium $[30,54]$, and $\beta$-catenin is involved in such mechanisms [29].

In the present study, cytoplasmic $\beta$-catenin was found to vary in intensity and pattern. These temporal variations seemed associated to sex steroid peripheral levels. Decreased cytoplasmic intensity was detected in early diestrus and in diestrus, when compared to the other cycle stages. The most frequent cytoplasmic pattern found was the diffuse type that has been observed in all stages except of estrus, where a supra-nuclear immunolabeling pattern predominated. This pattern could correspond to the cytosolic pool of inactive $\beta$ catenin that does not interact with either cadherin or the Wnt pathway [29]. Among all other stages, estrus displayed the strongest immunolabeling, associated with supra-nuclear 
location. Such a strong supra-nuclear immunolabeling might be associated with a transitory stimulation of the Wnt signaling pathway, in non-pregnant endometrium, as it clearly contrasts to the usual $\beta$-catenin cytoplasmic immunostaining. As a consequence, the Wntassociated pathways, demonstrated in humans to be dependent on high levels of estrogens and on cell proliferation [30], might also be activated in the canine endometrium during estrus.

In conclusion, this study showed a gradient of the E-cadherin immunolabeling in the canine endometrium, with a weaker immunolabeling observed in the surface epithelium and a stronger labeling in the deep glandular epithelium. Furthermore, a marked decrease in the immunolabeling was observed in secretory stages, associated with high progesterone levels. A decrease in the membrane and cytoplasmic intensity of immunolabeling for $\beta$-catenin during the secretory stages, accompanying the reduction in E-cadherin immunostaining, was also found. We speculate that a weakening of the lateral intercellular connections during diestrus would favor embryo implantation. The cyclic changes in the cytoplasmic immunopattern for $\beta$-catenin may suggest a possible activation of the Wnt signaling pathway during the proliferative stages of the canine estrous cycle.

\section{Acknowledgements}

The authors thank Mrs. Ligia Lourenço (UTAD) and Mrs. Annika Rikberg for their expert technical assistance.

The Portuguese Science and Technology Foundation (FCT) sponsored this work under the Project PEst-OE/AGR/UI0772/2014, and granted Rita Payan Carreira with a sabbatical fellowship (SFRH/BSAB/938/2009).

\section{References}


[1] Barrau MD, Abel JH, Jr., Verhage HG, Tietz WJ, Jr. Development of the endometrium during the estrous cycle in the bitch. Am J Anat. 1975;142:47-65.

[2] Rahnama F, Thompson B, Steiner M, Shafiei F, Lobie PE, Mitchell MD. Epigenetic regulation of E-cadherin controls endometrial receptivity. Endocrinology. 2009;150:1466-72. [3] Aplin JD. Adhesion molecules in implantation. Rev Reprod. 1997;2:84-93.

[4] Salamonsen LA, Nie G, Hannan NJ, Dimitriadis E. Society for Reproductive Biology Founders' Lecture 2009. Preparing fertile soil: the importance of endometrial receptivity. Reprod Fertil Dev. 2009;21:923-34.

[5] Aplin JD. Embryo implantation: the molecular mechanism remains elusive. Reprod Biomed Online. 2006;13:833-9.

[6] Cavagna M, Mantese JC. Biomarkers of endometrial receptivity - A review. Placenta. 2003;24.

[7] Bauersachs S, Mitko K, Ulbrich SE, Blum H, Wolf E. Transcriptome studies of bovine endometrium reveal molecular profiles characteristic for specific stages of estrous cycle and early pregnancy. Exp Clin Endocrinol Diabetes. 2008;116:371-84.

[8] Singh H, Aplin JD. Adhesion molecules in endometrial epithelium: tissue integrity and embryo implantation. J Anat. 2009;215:3-13.

[9] Halbleib JM, Nelson WJ. Cadherins in development: cell adhesion, sorting, and tissue morphogenesis. Genes Dev. 2006;20:3199-214.

[10] van der Linden PJ, de Goeij AF, Dunselman GA, Erkens HW, Evers JL. Expression of cadherins and integrins in human endometrium throughout the menstrual cycle. Fertil Steril. 1995;63:1210-6.

[11] Fujimoto J, Ichigo S, Hori M, Tamaya T. Alteration of E-cadherin, alpha- and betacatenin mRNA expression in human uterine endometrium during the menstrual cycle.

Gynecol Endocrinol. 1996;10:187-91.

[12] Ryan PL, Baum DL, Lenhart JA, Ohleth KM, Bagnell CA. Expression of uterine and cervical epithelial cadherin during relaxin-induced growth in pigs. Reproduction.

2001;122:929-37.

[13] Satterfield MC, Dunlap KA, Hayashi K, Burghardt RC, Spencer TE, Bazer FW. Tight and adherens junctions in the ovine uterus: differential regulation by pregnancy and progesterone. Endocrinology. 2007;148:3922-31.

[14] Kiewisz J, Kaczmarek MM, Andronowska A, Blitek A, Ziecik AJ. Gene expression of WNTs, $\beta$-catenin and $\mathrm{E}$-cadherin during the periimplantation period of pregnancy in pigs-involvement of steroid hormones. Theriogenology. 2011;76:687-99.

[15] Leckband D. Beyond structure: mechanism and dynamics of intercellular adhesion. Biochem Soc Trans. 2008;36:213-20. 
[16] Wirtz-Peitz F, Zallen JA. Junctional trafficking and epithelial morphogenesis. Current Opinion in Genetics and Development. 2009;19:350-6.

[17] Nejsum LN, Nelson WJ. A molecular mechanism directly linking E-cadherin adhesion to initiation of epithelial cell surface polarity. J Cell Biol. 2007;178:323-35.

[18] Li Q, Wang J, Armant DR, Bagchi MK, Bagchi IC. Calcitonin down-regulates E-cadherin expression in rodent uterine epithelium during implantation. J Biol Chem. 2002;277:4644755.

[19] Jha RK, Titus S, Saxena D, Kumar PG, Laloraya M. Profiling of E-cadherin, betacatenin and $\mathrm{Ca}(2+)$ in embryo-uterine interactions at implantation. FEBS Lett. 2006;580:5653-60.

[20] Maretzky T, Reiss K, Ludwig A, Buchholz J, Scholz F, Proksch E, et al. ADAM10 mediates E-cadherin shedding and regulates epithelial cell-cell adhesion, migration, and beta-catenin translocation. Proc Natl Acad Sci U S A. 2005;102:9182-7.

[21] Perry JK, Lins RJ, Lobie PE, Mitchell MD. Regulation of invasive growth: similar epigenetic mechanisms underpin tumour progression and implantation in human pregnancy. Clin Sci (Lond). 2010;118:451-7.

[22] Dawood MY, Lau M, Khan-Dawood FS. E-cadherin and its messenger ribonucleic acid in periimplantation phase human endometrium in normal and clomiphene-treated cycles. Am J Obstet Gynecol. 1998;178:996-1001.

[23] Liu G, Zhang X, Lin H, Wang H, Li Q, Ni J, et al. Effects of E-cadherin on mouse embryo implantation and expression of matrix metalloproteinase-2 and -9. Biochem Biophys Res Commun. 2006;343:832-8.

[24] Guo B, Han BC, Tian Z, Zhang XM, Jiang LX, Liu JX, et al. Expression and Hormonal Regulation of E-Cadherin in Canine Uterus During Early Pregnancy. Reprod Domest Anim. 2009.

[25] Lilien J, Balsamo J, Arregui C, Xu G. Turn-off, drop-out: functional state switching of cadherins. Dev Dyn. 2002;224:18-29.

[26] Nelson WJ. Regulation of cell-cell adhesion by the cadherin-catenin complex. Biochem Soc Trans. 2008;36:149-55.

[27] Yang S, Guo X, Debnath G, Mohandas N, An X. Protein 4.1R links E-cadherin/ $\mathbf{I}^{2}$-catenin complex to the cytoskeleton through its direct interaction with $\hat{i}^{2}$-catenin and modulates adherens junction integrity. Biochimica et Biophysica Acta - Biomembranes. 2009;1788:1458-65.

[28] Huber AH, Weis WI. The structure of the beta-catenin/E-cadherin complex and the molecular basis of diverse ligand recognition by beta-catenin. Cell. 2001;105:391-402. [29] Gottardi CJ, Gumbiner BM. Distinct molecular forms of beta-catenin are targeted to adhesive or transcriptional complexes. J Cell Biol. 2004;167:339-49. 
[30] Wang Y, Hanifi-Moghaddam P, Hanekamp EE, Kloosterboer HJ, Franken P, Veldscholte J, et al. Progesterone inhibition of Wnt/beta-catenin signaling in normal endometrium and endometrial cancer. Clin Cancer Res. 2009;15:5784-93.

[31] Chen Q, Zhang Y, Lu J, Wang Q, Wang S, Cao Y, et al. Embryo-uterine cross-talk during implantation: the role of Wnt signaling. Mol Hum Reprod. 2009;15:215-21.

[32] Wooding P, Burton G. Endotheliochorial Placentation: Cat, Dog, Bat. Comparative placentation : structures, functions and evolution. Heidelberg, Germany: Springer-Verlag Berlin Heidelberg; 2008. p. 169-83.

[33] Matsuzaki S, Darcha C, Maleysson E, Canis M, Mage G. Impaired down-regulation of E-cadherin and beta-catenin protein expression in endometrial epithelial cells in the midsecretory endometrium of infertile patients with endometriosis. J Clin Endocrinol Metab. 2010;95:3437-45.

[34] Birchmeier W, Behrens J. Cadherin Expression in Carcinomas - Role in the Formation of Cell-Junctions and the Prevention of Invasiveness. Biochimica Et Biophysica ActaReviews on Cancer. 1994;1198:11-26.

[35] Bremnes RM, Veve R, Hirsch FR, Franklin WA. The E-cadherin cell-cell adhesion complex and lung cancer invasion, metastasis, and prognosis. Lung Cancer. 2002;36:11524.

[36] Johnston. Disordres of the canine uterus and uterine tubes (oviducts). In: Johnston SD, Root Kustritz MV, Olson PS, editors. Canine and Feline Theriogenology. 1st ed.

Philadelphia: Saunders; 2001. p. 206-24.

[37] Shih HC, Shiozawa T, Miyamoto T, Kashima H, Feng YZ, Kurai M, et al. Immunohistochemical expression of E-cadherin and beta-catenin in the normal and malignant human endometrium: an inverse correlation between E-cadherin and nuclear beta-catenin expression. Anticancer Res. 2004;24:3843-50.

[38] Concannon PW. Reproductive cycles of the domestic bitch. Anim Reprod Sci. 2011;124:200-10.

[39] Barrau MD, Abel JH, Torbit CA, Tietz WJ. Development of the implantation chamber in the pregnant bitch. Am J Anat. 1975;143:115-30.

[40] Holst PA, Phemister RD. The prenatal development of the dog: preimplantation events. Biol Reprod. 1971;5:194-206.

[41] Payan-Carreira R, Pires MA, Holst BS, Rodriguez-Martinez H. Tumour Necrosis Factor in the Canine Endometrium: An Immunohistochemical Study. Reproduction in Domestic Animals. 2011;46:410-8.

[42] Payan-Carreira R, Santos C, Miranda S, Pereira RMLN, Santos D, Pires MA. Temporal changes in neutral endopeptidase/CD10 immunoexpression in the cyclic and early pregnant canine endometrium. Theriogenology. 2014;82:815-26. 
[43] Amoroso EC. Placentation. In: Parkes AS, editor. Marshall's Physiology of Reproduction. London: Longmans Green and Co.; 1952. p. 127-311.

[44] Gama A, Paredes J, Gartner F, Alves A, Schmitt F. Expression of E-cadherin, Pcadherin and beta-catenin in canine malignant mammary tumours in relation to clinicopathological parameters, proliferation and survival. Vet J. 2008;177:45-53.

[45] McEwan M, Lins RJ, Munro SK, Vincent ZL, Ponnampalam AP, Mitchell MD. Cytokine regulation during the formation of the fetal-maternal interface: focus on cell-cell adhesion and remodelling of the extra-cellular matrix. Cytokine Growth Factor Rev. 2009;20:241-9. [46] Jeong JW, Lee HS, Franco HL, Broaddus RR, Taketo MM, Tsai SY, et al. beta-catenin mediates glandular formation and dysregulation of beta-catenin induces hyperplasia formation in the murine uterus. Oncogene. 2009;28:31-40.

[47] Bartley J, Jülicher A, Hotz B, Mechsner S, Hotz H. Epithelial to mesenchymal transition (EMT) seems to be regulated differently in endometriosis and the endometrium. Arch Gynecol Obstet. 2014;289:871-81.

[48] Yoshida R, Kimura N, Harada Y, Ohuchi N. The loss of E-cadherin, alpha- and betacatenin expression is associated with metastasis and poor prognosis in invasive breast cancer. Int J Oncol. 2001;18:513-20.

[49] Delva E, Kowalczyk AP. Regulation of cadherin trafficking. Traffic. 2009;10:259-67. [50] Lu M, Marsters S, Ye X, Luis E, Gonzalez L, Ashkenazi A. E-cadherin couples death receptors to the cytoskeleton to regulate apoptosis. Mol Cell. 2014;54:987-98.

[51] Gallegos LL, Brugge JS. Live free or die: cell-cell adhesion regulates sensitivity to trailinduced apoptosis. Dev Cell. 2014;30:3-4.

[52] Ferreira AC, Suriano G, Mendes N, Gomes B, Wen X, Carneiro F, et al. E-cadherin impairment increases cell survival through Notch-dependent upregulation of $\mathrm{Bcl}-2$. Hum $\mathrm{Mol}$ Genet. 2012;21:334-43.

[53] Ishiguro K, Baba E, Torii R, Tamada H, Kawate N, Hatoya S, et al. Reduction of mucin1 gene expression associated with increased Escherichia coli adherence in the canine uterus in the early stage of dioestrus. Vet J. 2007;173:325-32.

[54] Nei H, Saito T, Yamasaki H, Mizumoto H, Ito E, Kudo R. Nuclear localization of betacatenin in normal and carcinogenic endometrium. Mol Carcinog. 1999;25:207-18. 


\section{Tables}

Table 1 - E-cadherin and $\beta$-catenin membrane immunoreactivity scores in the canine endometrium throughout the stages of the estrous cycle. Numbers in the table represent to the number of samples.

\begin{tabular}{|c|c|c|c|c|c|c|c|c|c|c|c|c|c|}
\hline & \multirow[b]{2}{*}{ Stages } & \multicolumn{4}{|c|}{ SE } & \multicolumn{4}{|c|}{ SGE } & \multicolumn{4}{|c|}{ DGE } \\
\hline & & 1 & 2 & 3 & 4 & 1 & 2 & 3 & 4 & 1 & 2 & 3 & 4 \\
\hline \multirow{5}{*}{ E-Cadherin } & Anestrus & 0 & 10 & 0 & 0 & 0 & 0 & 10 & 0 & 0 & 0 & 0 & 10 \\
\hline & Proestrus & 0 & 8 & 0 & 0 & 0 & 1 & 7 & 0 & 0 & 0 & 0 & 8 \\
\hline & Estrus & 1 & 9 & 0 & 0 & 0 & 5 & 5 & 0 & 0 & 0 & 5 & 5 \\
\hline & Early diestrus & 12 & 0 & 0 & 0 & 0 & 12 & 0 & 0 & 0 & 11 & 1 & 0 \\
\hline & Diestrus & 10 & 0 & 0 & 0 & 2 & 8 & 0 & 0 & 0 & 1 & 9 & 0 \\
\hline \multirow{5}{*}{$\beta$-catenin } & Anestrus & 8 & 2 & 0 & 0 & 3 & 7 & 0 & 0 & 0 & 10 & 0 & 0 \\
\hline & Proestrus & 7 & 1 & 0 & 0 & 3 & 5 & 0 & 0 & 1 & 7 & 0 & 0 \\
\hline & Estrus & 10 & 0 & 0 & 0 & 6 & 4 & 0 & 0 & 3 & 7 & 0 & 0 \\
\hline & Early diestrus & 11 & 1 & 0 & 0 & 8 & 4 & 0 & 0 & 3 & 9 & 0 & 0 \\
\hline & Diestrus & 9 & 1 & 0 & 0 & 4 & 6 & 0 & 0 & 0 & 10 & 0 & 0 \\
\hline
\end{tabular}

SE - surface endometrium; SGE - superficial glandular endometrium; DGE - deep glandular endometrium 
Table 2 - Cytoplasmic intensity and pattern of immunoreaction against $\beta$-catenin in the cyclic canine endometrium. Numbers in the table represent to the number of samples.

\begin{tabular}{|c|c|c|c|c|c|c|c|}
\hline & & Score & Anestrus & Proestrus & Estrus & Early Diestrus & Diestrus \\
\hline \multirow{12}{*}{$\begin{array}{l}\text { Intensity } \\
\text { score }\end{array}$} & \multirow{4}{*}{ SE } & 1 & 2 & 1 & 2 & 11 & 10 \\
\hline & & 2 & 8 & 7 & 8 & 1 & 0 \\
\hline & & 3 & 0 & 0 & 0 & 0 & 0 \\
\hline & & 4 & 0 & 0 & 0 & 0 & 0 \\
\hline & \multirow{4}{*}{ SGE } & 1 & 1 & 2 & 1 & 5 & 8 \\
\hline & & 2 & 9 & 6 & 9 & 7 & 2 \\
\hline & & 3 & 0 & 0 & 0 & 0 & 0 \\
\hline & & 4 & 0 & 0 & 0 & 0 & 0 \\
\hline & \multirow{4}{*}{ DGE } & 1 & 0 & 0 & 0 & 0 & 1 \\
\hline & & 2 & 8 & 8 & 2 & 9 & 9 \\
\hline & & 3 & 2 & 0 & 8 & 3 & 0 \\
\hline & & 4 & 0 & 0 & 0 & 0 & 0 \\
\hline \multirow{12}{*}{ Pattern } & \multirow{4}{*}{ SE } & diffuse & 9 & 4 & 2 & 8 & 6 \\
\hline & & apical & 1 & 4 & 2 & 3 & 4 \\
\hline & & scattered & 0 & 0 & 0 & 0 & 0 \\
\hline & & supranuclear & 0 & 0 & 6 & 1 & 0 \\
\hline & \multirow{4}{*}{ SGE } & diffuse & 10 & 5 & 0 & 1 & 4 \\
\hline & & apical & 0 & 3 & 0 & 3 & 4 \\
\hline & & scattered & 0 & 0 & 2 & 6 & 1 \\
\hline & & supranuclear & 0 & 0 & 8 & 2 & 1 \\
\hline & \multirow{4}{*}{ DGE } & diffuse & 10 & 8 & 2 & 8 & 6 \\
\hline & & apical & 0 & 0 & 0 & 1 & 4 \\
\hline & & scattered & 0 & 0 & 0 & 1 & 0 \\
\hline & & supranuclear & 0 & 0 & 8 & 2 & 0 \\
\hline
\end{tabular}

SE - surface endometrium; SGE - superficial glandular endometrium; DGE - deep glandular endometrium 
Table 3 - E-Cadherin and $\beta$-Catenin membrane immunoreactivity scores in early pregnant canine endometrium (pregnancy days 17 to 20; $n=9$ ). Numbers in the table represent to the number of samples.

\begin{tabular}{|c|c|c|c|c|c|c|c|}
\hline \multirow{2}{*}{ Marker } & \multirow{2}{*}{ Scores } & \multirow{2}{*}{ Trophoblast } & \multirow{2}{*}{ SE } & \multicolumn{2}{|c|}{ Labyrinth epithelium } & \multirow{2}{*}{ Lac E } & \multirow{2}{*}{ DGE } \\
\hline & & & & Trophoblast & Decidual cells & & \\
\hline \multirow{4}{*}{$\begin{array}{c}\text { E- } \\
\text { Cadherin }\end{array}$} & 1 & 0 & 5 & 0 & 9 & 0 & 2 \\
\hline & 2 & 0 & 1 & 3 & 0 & 0 & 6 \\
\hline & 3 & 5 & 0 & 6 & 0 & 4 & 1 \\
\hline & 4 & 4 & 0 & 0 & 0 & 0 & 0 \\
\hline \multirow{4}{*}{$\beta$-Catenin } & 1 & 6 & 1 & 6 & 0 & 1 & 3 \\
\hline & 2 & 3 & 5 & 3 & 0 & 3 & 5 \\
\hline & 3 & 0 & 0 & 0 & 0 & 0 & 1 \\
\hline & 4 & 0 & 0 & 0 & 0 & 0 & 0 \\
\hline
\end{tabular}

SE - surface endometrium; DGE - deep glandular endometrium; Lac E - Lacunar epithelium. 


\section{Figures}

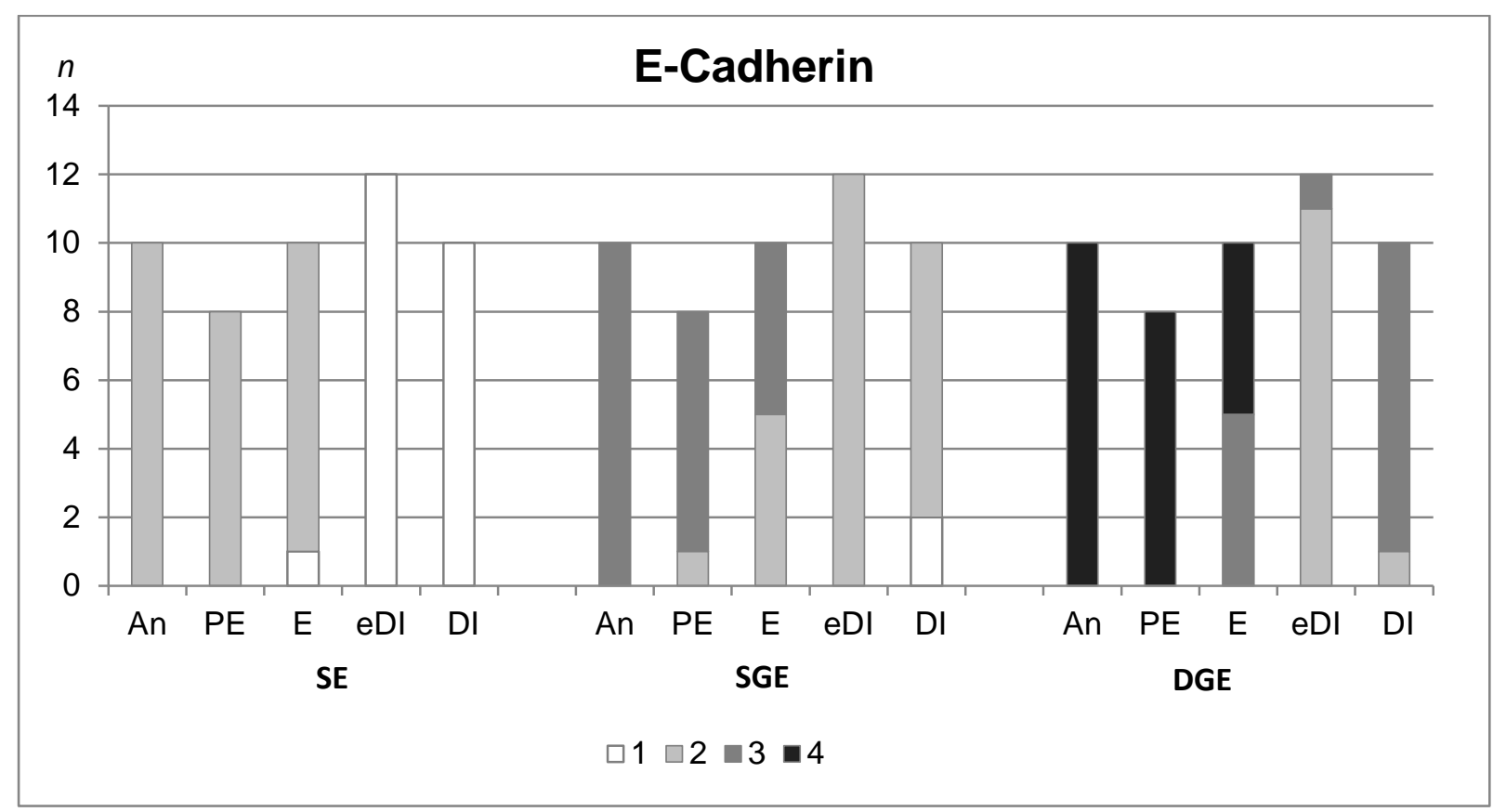

Figure 1 - Graphic representation of the endometrial membrane intensity scoring for Ecadherin during the canine estrous cycle: proestrus $(n=8)$, estrus $(n=10)$, early diestrus $(n=12)$, full diestrus $(n=10)$ and anestrus $(n=10)$

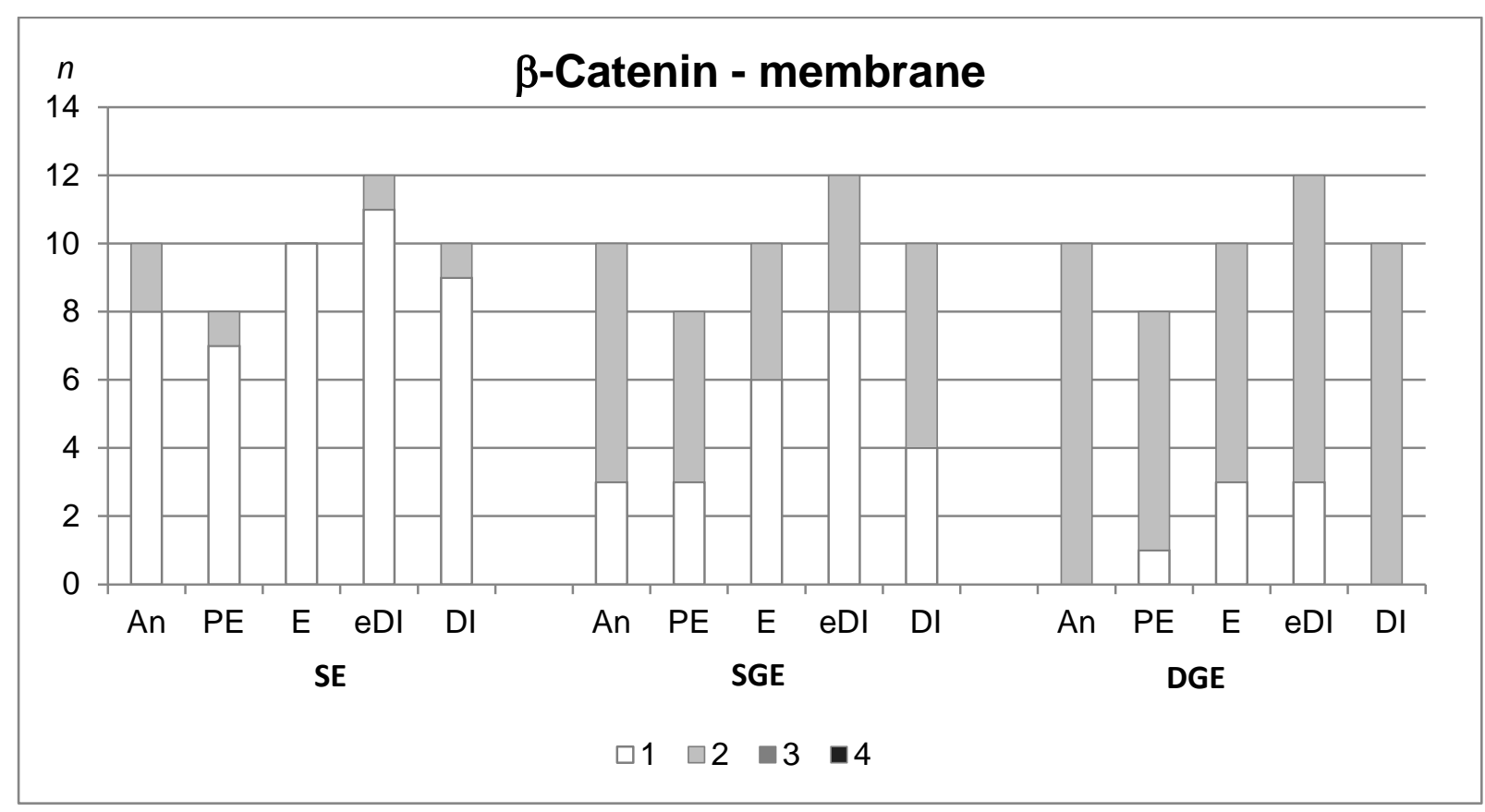

Figure 2 - Graphic representation of the endometrial membrane intensity scoring for $\beta$ catenin throughout the canine estrous cycle: proestrus $(n=8)$, estrus $(n=10)$, early diestrus $(n=12)$, full diestrus $(n=10)$ and anestrus $(n=10)$ 

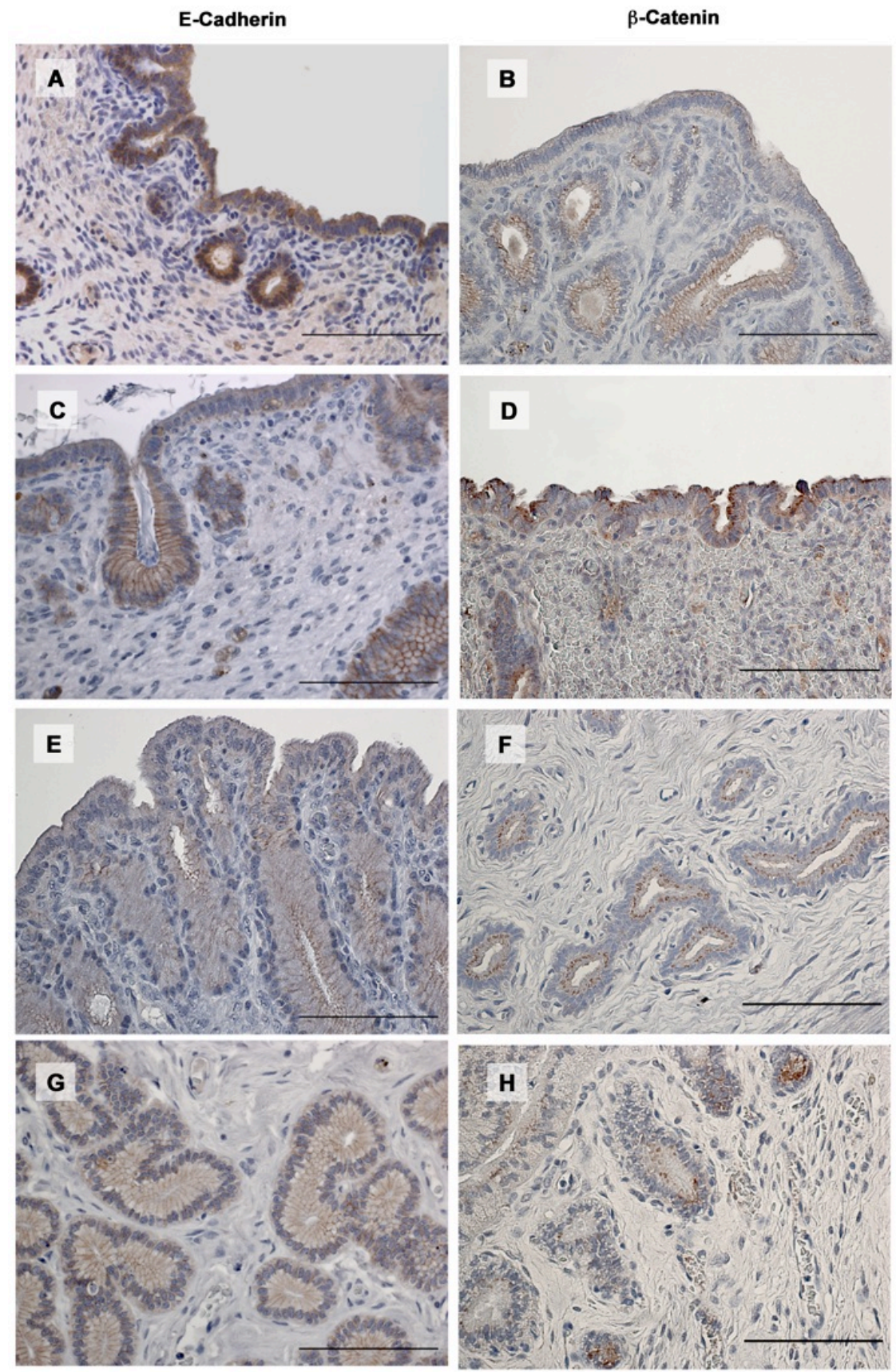

Figure 3 - Immunohistochemical expression of E-cadherin and $\beta$-catenin in the normal canine endometrium throughout the estrous cycle (bar: $100 \mu \mathrm{m})$. In the plansch, the left column of images corresponds to the membranar immunoreaction against $E$-cadherin in the superficial endometrial layers in proestrus (A), estrus (C) and early diestrus (E) or to DGE immunolabeling in full diestrus $(G)$, while the column in the right depicts the diverse patterns associated with $\beta$-catenin immunolabeling: the membranar pattern $(B)$, here observed in the SE and SGE in full diestrus samples; the apical cytoplasmic pattern in the SE of a proestrus sample (D); the supranuclear cytoplasmic pattern prevailing in estrus samples (F); and the scattered cytoplasmic pattern evidenced in some early diestrus samples $(H)$. 


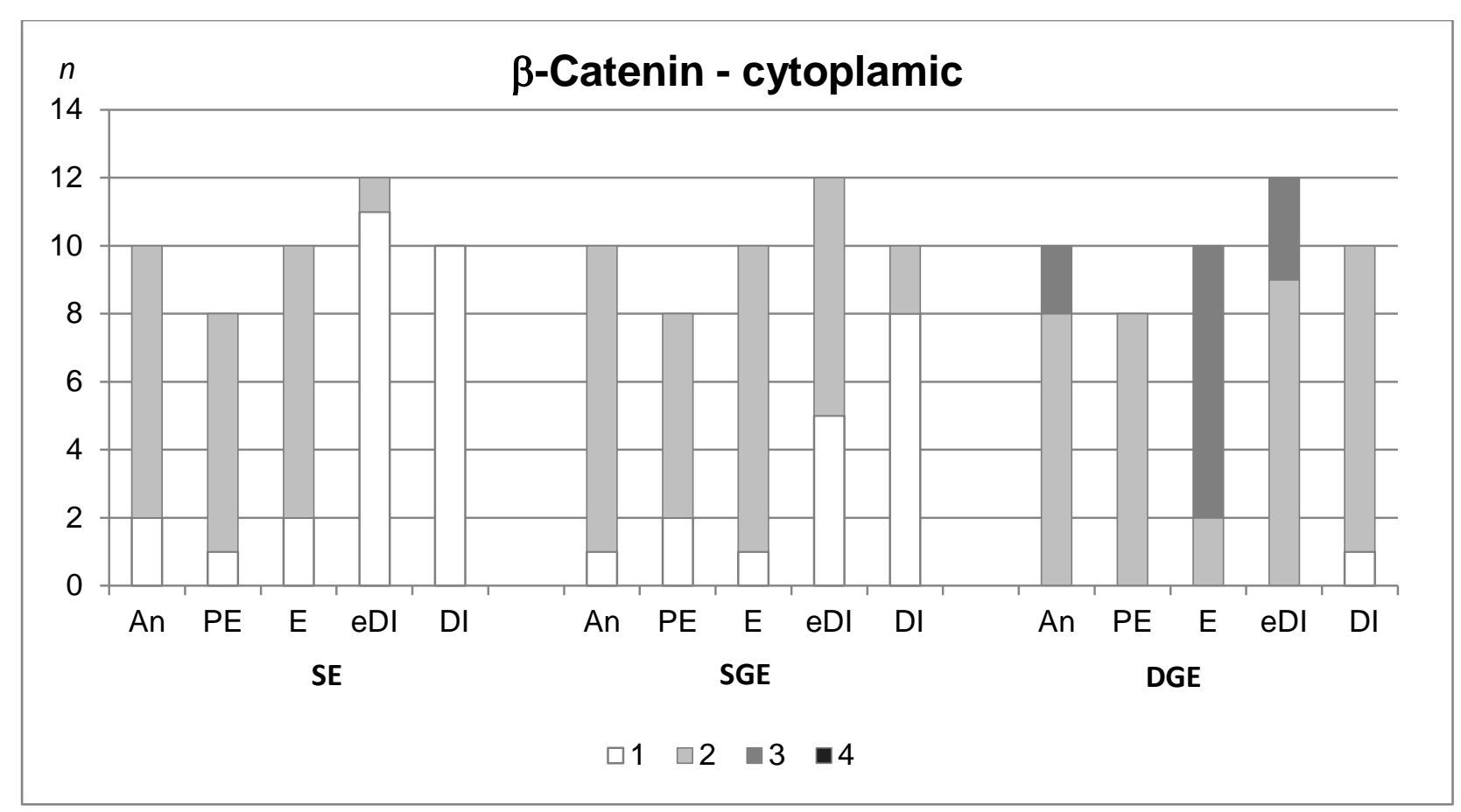

Figure 4 - Graphic representation of the endometrial cytoplasmic intensity scoring for $\beta$ catenin during the canine estrous cycle: proestrus $(n=8)$, estrus $(n=10)$, early diestrus $(n=12)$, full diestrus $(n=10)$ and anestrus $(n=10)$

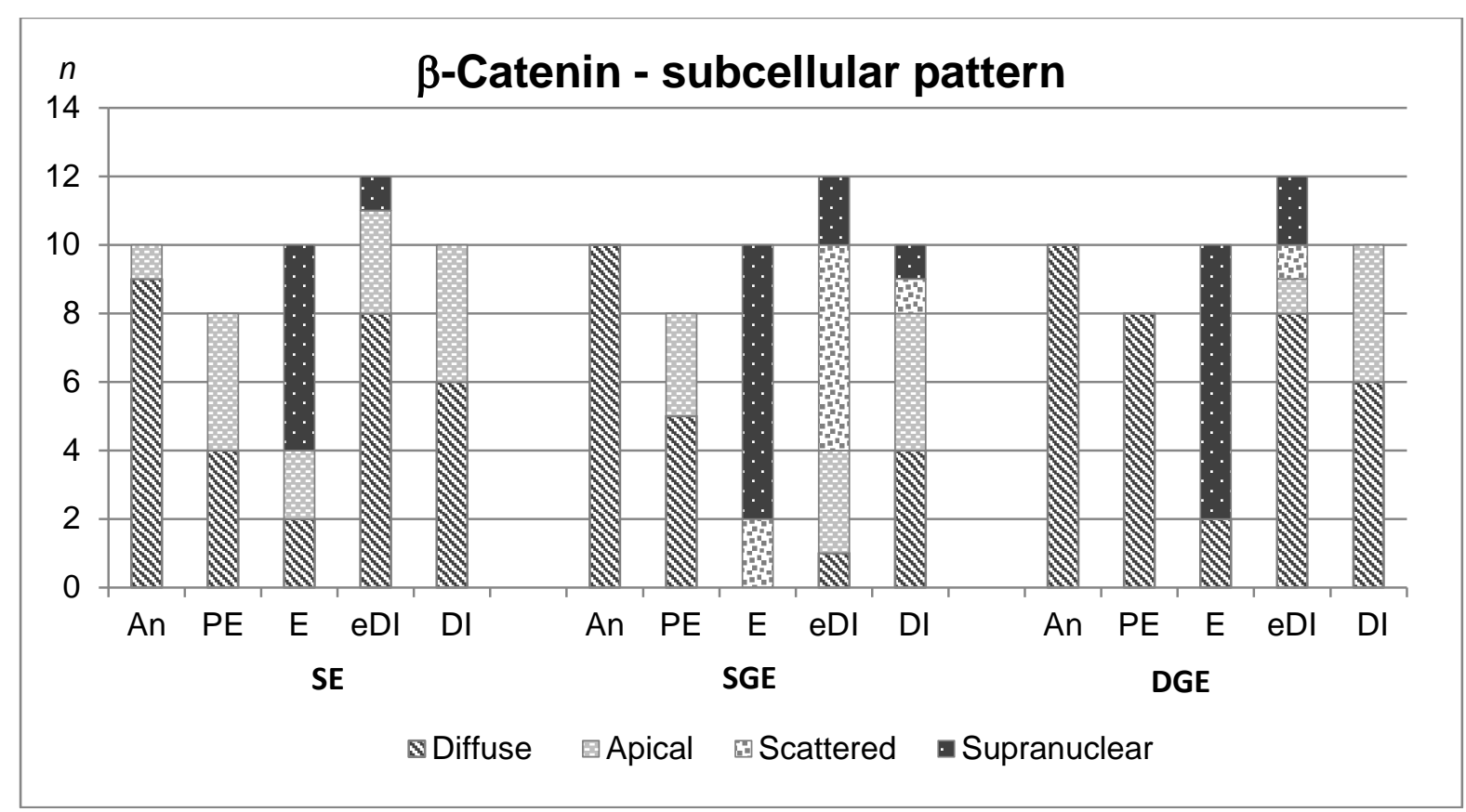

Figure 5 - Graphic representation of the cytoplasmic pattern of immunoreaction for $\beta$ catenin throughout the canine estrous cycle: proestrus $(n=8)$, estrus $(n=10)$, early diestrus $(n=12)$, full diestrus $(n=10)$ and anestrus $(n=10)$ 

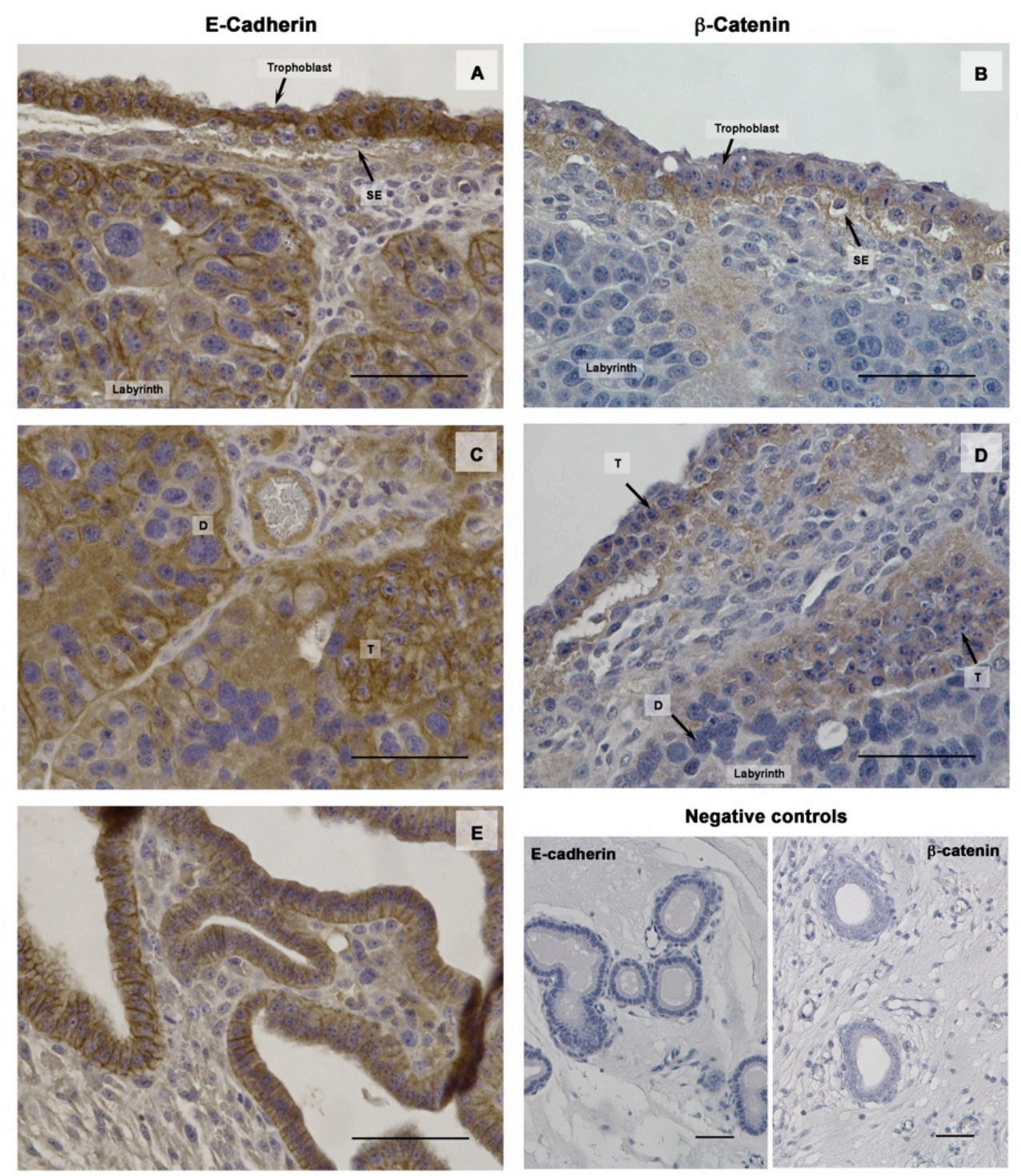

Figure 6 - Immunohistochemical expression of E-cadherin and $\beta$-catenin in endometrium from early pregnancy (days 17 to 20) (bar: $50 \mu \mathrm{m}$ ). In the plansch, images in the left column correspond to the immunoreaction against E-cadherin during the early steps of endometrial invasion: At the endometrial surface $(A)$, the trophoblast, showing a strong intensity score, lined the SE that does not show a membrane reaction for E-Cadherin. In the labyrinth (C), trophoblast cells showed a strong membrane pattern contrasting to the absent E-cadherin membrane immunolabeling shown by decidual cells. In lacunae (E), the epithelial cells showed strong membrane immunoscoring. The right column represents the $\beta$-catenin immunolabeling. (B) Weak to moderate membranous intensity score was evidenced in the trophoblast, while the SE displayed a moderate cytoplasmic pattern, no membrane pattern being discernible. In the labyrinth ( $B$ and $D$ ) a moderate intensity of immunostaining, either membrane or cytoplasmic, was observed in the trophoblast, in contrast to the negative decidual cells. The images on the right bottom represent the negative controls for E-cadherin and $\beta$-catenin, respectively. T - Trophoblast; Decidual cells (D). 